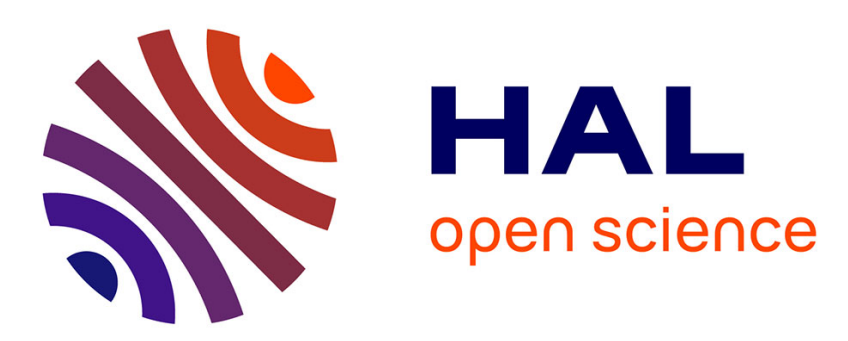

\title{
Multiple timescale and sensitivity analysis for the passive control of the cylinder flow
}

Olivier Marquet, Denis Sipp, Laurent Jacquin, Jean-Marc Chomaz

\section{To cite this version:}

Olivier Marquet, Denis Sipp, Laurent Jacquin, Jean-Marc Chomaz. Multiple timescale and sensitivity analysis for the passive control of the cylinder flow. 5th AIAA Theoretical Fluid Mechanics Conference, Jun 2008, Seattle, France. pp.AIAA 2008-4228, 10.2514/6.2008-4228 . hal-01026007

\section{HAL Id: hal-01026007 \\ https://hal-polytechnique.archives-ouvertes.fr/hal-01026007}

Submitted on 7 Dec 2017

HAL is a multi-disciplinary open access archive for the deposit and dissemination of scientific research documents, whether they are published or not. The documents may come from teaching and research institutions in France or abroad, or from public or private research centers.
L'archive ouverte pluridisciplinaire HAL, est destinée au dépôt et à la diffusion de documents scientifiques de niveau recherche, publiés ou non, émanant des établissements d'enseignement et de recherche français ou étrangers, des laboratoires publics ou privés. 


\title{
Multiple timescale and sensitivity analysis for the passive control of the cylinder flow
}

\author{
O. Marquet \\ Universität der Bundeswehr (UniBwM), München, 85577, Germany \\ D. Sipp, L. Jacquin \\ Department of Fundamental and Experimental Aerodynamics - ONERA, Meudon, 92190, France \\ J.-M. Chomaz \\ Laboratoire d'Hydrodynamique (LadHyx) - Ecole Polytechnique, Palaiseau, 91128, France
}

\begin{abstract}
A theoretical approach is developed to predict the effect of a passive control device on the global stability of a cylinder flow close to the critical Reynolds number $\boldsymbol{R e}_{c}=46.8$. The passive control device is here a small control cylinder that is modelled by a local force. This force is the sum of a steady component, acting on the base-flow, and of an unsteady component, acting at the perturbation level. To understand how these two components affect the flow stability, a multiple timescale analysis is first performed at the bifurcation. The leading global mode responsible for the onset of the Von Karman street is assumed to oscillate on a "fast" timescale and its amplitude to grow on a "slow" timescale. Then a sensitivity analysis of the leading global eigenvalue with respect to the steady and unsteady components of the force is developed. By combining these two analysis, one obtains maps of the growth rate and pulsation of the perturbation as a function of the position of the local force. The regions of the flow where the placement of a control cylinder suppresses the Von Karman instability are very well reproduced.
\end{abstract}

\section{Introduction}

Control of vortex shedding behind a circular cylinder has received much attention in the past and various passive and active control techniques have been tested on this flow both experimentally and numerically. Concerning passive control, Strykowski \& Sreenivasan $(1990)^{7}$ have experimentally investigated how a small control cylinder suitably placed in the wake of the main cylinder could alter the vortex shedding. For various diameter ratios of the two cylinders they determined the regions of the flow where the placement of the control cylinder leads to a complete suppression of the phenomenon over a specific range of Reynolds numbers. They also provided experimental evidence linking vortex shedding to the onset of a global instability, and the effect of the adequately positioned control cylinder to a damping of this instability. A theoretical approach of such a passive control has first been developed by Hill $(1992)^{4}$ but this study is remained confidential for many years. Recently Giannetti \& Luchini $(2003,2007)^{2,3}$ and Chomaz $(2005)^{1}$ have reconsidered this problem using modern tools to analyse the stability of non-parallel flows. In particular, they have shown how direct and adjoint global modes could be used to identify the region of the flow at the origin of the instability. Their analysis may be extrapolated to a passive control study if one considers that introducing a small device in the flow can be modeled by an unsteady local force acting at the perturbation level. More recently Marquet, Sipp \& Jacquin $(2008)^{5}$ have modeled the presence of a control cylinder in the flow not by an unsteady force as did in Giannetti \& Luchini $(2007),{ }^{3}$ but rather by a steady force modifying the base-flow on which the perturbation develops. Their analysis is based on the introduction of a sensitivity function to a steady force. Good predictions of the stabilizing regions for the control cylinder have been obtained. However, from a physical point of view, the presence of control cylinder in the flow should be modeled by a force acting both on the base-flow and at the perturbation level. The objective of the present paper is to develop a theoretical framework in which

- the control cylinder is modeled as the sum of a steady and an unsteady force, 
- the effects of these steady and unsteady components onto the flow stability are both assessed.

To this end, a multiple timescale analysis is developed at the critical Reynolds number $R e_{c}$. It is based on the assumption that the leading global mode responsible for the onset of the vortex shedding oscillates on a "fast" timescale but that its complex amplitude grows on a "slow" timescale related to the introduction of the control cylinder. By combining it with a sensitivity analysis to a steady and an unsteady force, it is shown how the steady and unsteady components of the force that models the control cylinder modify the stability of the flow.

\section{Problem formulation}

The two-dimensional motion of a viscous fluid around a circular cylinder is described in terms of the velocity and pressure fields $(\mathbf{u}, p)$ governed by the unsteady incompressible Navier-Stokes equations

$$
\partial_{t} \mathbf{u}+\nabla \mathbf{u} \cdot \mathbf{u}+\nabla p-R e^{-1} \nabla^{2} \mathbf{u}=\mathbf{f}, \quad \nabla \cdot \mathbf{u}=0,
$$

where $R e$ is the Reynolds number based on the cylinder diameter $D=1$ and on the upstream velocity $\mathbf{u}_{\infty}=1$, and $\mathbf{f}$ is a forcing term in the momentum equations. This term vanishes when the flow is not controlled. The control device is a cylinder of diameter $d$ located at the position $\left(x_{f}, y_{f}\right)$ in the flow. It is called therein after the control cylinder. In the present paper, such a control device is not introduced into the physical flow but modeled in the Navier-Stokes equations by the forcing term $\mathbf{f}$.

\section{A. Modelisation of the control cylinder}

The control cylinder is modeled by a local force $\mathbf{f}$ which is the opposite of the drag force filled by the control cylinder. Its expression is

$$
\mathbf{f}(x, y)=-\frac{1}{2} d C_{D}\left(R e_{f}\right)\|\mathbf{u}\| \mathbf{u} \delta\left(x-x_{f}, y-y_{f}\right)
$$

where $\left(x_{f}, y_{f}\right)$ denotes the position of the control cylinder, $d$ is the control cylinder diameter, $C_{D}$ stands for the drag coefficient and $\delta$ is the Dirac function. The drag coefficient depends on the Reynolds number $R e_{f}=\left\|\mathbf{u}_{f}\right\| d R e$ based on the velocity $\mathbf{u}_{f}=\mathbf{u}\left(x_{f}, y_{f}\right)$ and the control cylinder diameter $d$. An analytical expression of the drag coefficient $C_{D}\left(R e_{f}\right)$ depends on the range of values of local Reynolds number $R e_{f}$. For $d=0.1$, this Reynolds number $R e_{f}$ extents in the range [1,10]. A good analytical expression for the drag coefficient is given by

$$
C_{D}(x)=\frac{1}{x(a+b \ln x)}, \text { with } a=0.0987, b=-0.0627
$$

\section{B. Multiple timescale analysis}

We consider the two small parameters

$$
\epsilon=d C_{D}\left(R e_{d}\right) \ll 1 \text { and } \alpha \ll 1 .
$$

The first small parameter, denoted $\epsilon$, is introduced to take into account the presence of the control cylinder. The drag coefficient $C_{D}$ of the model force is now a function of the local Reynolds number $R e_{d}=$ $d\left\|\mathbf{u}_{\mathbf{0 0}}\left(x_{f}, y_{f}\right)\right\| R e_{c}$, based on the control cylinder diameter $d$ and on the velocity $\mathbf{u}_{\mathbf{0 o}}$ measured at the station $\left(x_{f}, y_{f}\right)$ where the control cylinder is introduced. In the following it will be shown that this is the velocity of the unperturbed base-flow at the cirtical Reynolds number $R e_{c}$. The second parameter, denoted $\alpha$, will stand for the amplitude of the perturbation developing on this base flow. In order these two parameters to be small, the control cylinder diameter should be small, i.e. $d \ll 1$, as well as the amplitude of the perturbation. The present analysis is therefore restricted to describe the influence of small control cylinder on the flow computed at the critical Reynolds number $R e_{c}$.

One performs a multiple timescale analysis based on the "fast" timescale $t$ and the "slow" timescale $t_{1}=\epsilon t$. The following asymptotic expansion of the flowfield $\mathbf{U}=(\mathbf{u}, p)^{T}$ with the small parameter $\epsilon$ and $\alpha$ is considered:

$$
\mathbf{U}=\mathbf{U}_{\mathbf{0 0}}+\alpha^{1} \epsilon^{0} \mathbf{U}_{\mathbf{1 0}}+\alpha^{0} \epsilon^{1} \mathbf{U}_{\mathbf{0 1}}+\alpha^{1} \epsilon^{1} \mathbf{U}_{\mathbf{1 1}}+\ldots
$$


This expansion is first introduced into the expression of the model local force. At leading orders in $\epsilon, \alpha$ one obtains

$$
\begin{aligned}
\mathbf{f}=\alpha^{0} \epsilon^{1} \mathbf{f}_{\mathbf{s}}+\alpha^{1} \epsilon^{1} \mathbf{f}_{\mathbf{u}}+\ldots, \text { with } \mathbf{f}_{\mathbf{s}} & =-\frac{1}{2}\left\|\mathbf{u}_{\mathbf{0 0}}\right\| \mathbf{u}_{\mathbf{0 0}} \delta\left(x-x_{f}, y-y_{f}\right) \\
\mathbf{f}_{\mathbf{u}} & =-\frac{1}{2}\left(\frac{\mathbf{u}_{\mathbf{0 0}} \cdot \mathbf{u}_{\mathbf{1 0}}}{\left\|\mathbf{u}_{\mathbf{0 0}}\right\|} \mathbf{u}_{\mathbf{0 0}}+\left\|\mathbf{u}_{\mathbf{0 0}}\right\| \mathbf{u}_{\mathbf{1 0}}\right) \delta\left(x-x_{f}, y-y_{f}\right)
\end{aligned}
$$

It will be shown that $\mathbf{f}_{\mathbf{s}}$ and $\mathbf{f}_{\mathbf{u}}$ are respectively the steady and unsteady components of the local force modeling the control cylinder. The expansion (5) is then introduced into the Navier-Stokes equations (1) for the critical Reynolds number $R e_{c}$. One obtains a series of equations at various orders in $\epsilon^{i} \alpha^{j}$, which are now detailed.

- At order $\alpha^{0} \epsilon^{0}$, one obtains that the base-flow flowfield $\mathbf{U}_{\mathbf{0 0}}=\left(\mathbf{u}_{\mathbf{0 0}}, p_{00}\right)^{T}$ is a solution of the steady Navier-Stokes equations at the critical Reynolds number $R e_{c}$, that read

$$
\nabla \mathbf{u}_{\mathbf{0 0}} \cdot \mathbf{u}_{\mathbf{0 0}}+\nabla \mathbf{u}_{\mathbf{0 0}} \cdot \mathbf{u}_{\mathbf{0 0}}+\nabla p_{00}-R e_{c}^{-1} \nabla^{2} \mathbf{u}_{\mathbf{0 0}}=\mathbf{0}, \quad \nabla \cdot \mathbf{u}_{\mathbf{0 0}}=0 .
$$

- At order $\alpha^{1} \epsilon^{0}$, one obtains that the perturbation flowfield $\mathbf{U}_{\mathbf{1 0}}=\left(\mathbf{u}_{\mathbf{1 0}}, p_{10}\right)^{T}$ is a solution of the following homogeneous equations

$$
\left(\partial_{t} \mathcal{L}+\mathcal{M}\right) \mathbf{U}_{10}=\mathbf{0}
$$

where $\mathcal{L}$ and $\mathcal{M}$ are two linear operators defined by

$$
\mathcal{L}=\left(\begin{array}{cc}
\mathcal{I} & 0 \\
0 & 0
\end{array}\right), \quad \mathcal{M}=\left(\begin{array}{cc}
\nabla() \cdot \mathbf{u}_{\mathbf{0 0}}+\nabla \mathbf{u}_{\mathbf{0 0}} \cdot()-R e_{c}^{-1} \Delta & \nabla \\
\nabla^{T} & 0
\end{array}\right)
$$

The perturbation $\mathbf{U}_{\mathbf{1 0}}$ is sought in the form of global normal modes

$$
\mathbf{U}_{10}=\frac{1}{2}\left(\hat{\mathbf{U}}_{\mathbf{1 0}} \exp [\sigma t]+c . c .\right)
$$

where $\sigma=\lambda+i \omega$ is a complex value, called eigenvalue, associated to a complex flowfield $\hat{\mathbf{U}}_{\mathbf{1 0}}=$ $\left(\hat{\mathbf{u}}_{10}, \hat{p}_{10}\right)^{T}$, called global mode. $\lambda$ and $\omega$ are respectively the growth rate and the pulsation of the global mode. Introducing the above decomposition into the equations (7), one obtains that the pair $\left(\sigma ; \hat{\mathbf{U}}_{\mathbf{1 0}}\right)$ is a solution of the generalized eigenvalue problem:

$$
(\sigma \mathcal{L}+\mathcal{M}) \hat{\mathbf{U}}_{10}=\mathbf{0}
$$

Solving this problem shows that there exists a global mode $\hat{\mathbf{U}}_{\mathbf{1 0}}^{\mathrm{c}}$ which is marginally stable, i.e. $\sigma_{c}=$ $0+i \omega_{c}$. The pair $\left(\sigma_{c}, \hat{\mathbf{U}}_{\mathbf{1 0}}^{\mathbf{c}}\right)$ is therefore solution of

$$
\left(i \omega_{c} \mathcal{L}+\mathcal{M}\right) \hat{\mathbf{U}}_{\mathbf{1 0}}^{\mathbf{c}}=\mathbf{0}
$$

Now, the perturbation is chosen in the form

$$
\mathbf{U}_{\mathbf{1 0}}=\frac{1}{2}\left(A\left(t_{1}\right) \hat{\mathbf{U}}_{\mathbf{1 0}}^{\mathbf{c}} \exp \left[i \omega_{c} t\right]+c . c .\right)
$$

where $A$ is the complex amplitude of the perturbation that depends on the slow-time scales $t_{1}=\epsilon t$.

- At order $\alpha^{0} \epsilon^{1}$, one obtains that the flowfield $\mathbf{U}_{\mathbf{0 1}}=\left(\mathbf{u}_{\mathbf{0 1}}, p_{01}\right)^{T}$ is a solution of the equations

$$
\left(\partial_{t} \mathcal{L}+\mathcal{M}\right) \mathbf{U}_{\mathbf{0 1}}=\mathbf{F}_{\mathbf{s}}
$$

where the term on the right hand-side $\mathbf{F}_{\mathbf{s}}=\left(\mathbf{f}_{\mathbf{s}}, 0\right)^{T}$ is a forcing term, and the forcing term in the momentum equations $\mathbf{f}_{\mathbf{s}}$ reads

$$
\mathbf{f}_{\mathbf{s}}(x, y)=-\frac{1}{2}\left\|\mathbf{u}_{\mathbf{0 0}}\right\| \mathbf{u}_{\mathbf{0 0}} \delta\left(x-x_{f}, y-y_{f}\right)
$$

Since the base-flow velocity $\mathbf{u}_{\mathbf{0 0}}$ is steady, this forcing term is the steady component of the force modeling the presence of the control cylinder. Therefore, $\mathbf{U}_{\mathbf{0 1}}$ is the base-flow modification induced by the presence of the control cylinder and is solution of the steady equation

$$
\mathcal{M} \mathbf{U}_{01}=\mathbf{F}_{01}
$$


- At order $\alpha^{1} \epsilon^{1}$, one obtains that $\mathbf{U}_{\mathbf{1 1}}=\left(\mathbf{u}_{\mathbf{1 1}}, p_{11}\right)^{T}$ is solution of the equation

$$
\left(\partial_{t} \mathcal{L}+\mathcal{M}\right) \mathbf{U}_{\mathbf{1 1}}=\frac{1}{2}\left(\left(-\frac{\partial A}{\partial t_{1}} \mathcal{L} \hat{\mathbf{U}}_{\mathbf{1 0}}^{\mathbf{c}}+A \hat{\mathbf{F}}_{\mathbf{1 1}}\right) \exp \left[i \omega_{c} t\right]+\text { c.c. }\right)
$$

where two forcing terms oscillating at the pulsation $\omega_{c}$ appear on the right-hand side of the equation. The first one results from the decomposition of the perturbation (see Eq. 12) in which the amplitude $A$ depends on the slow-time scale $t_{1}=\epsilon t$. The second one, denoted $\hat{\mathbf{F}}_{\mathbf{1 1}}$, reads

$$
\hat{\mathbf{F}}_{\mathbf{1 1}}=\hat{\mathbf{F}}_{\mathbf{u}}+\hat{\mathbf{F}}_{\mathbf{i}}=\left(\begin{array}{c}
\hat{\mathbf{f}}_{\mathbf{u}} \\
0
\end{array}\right)+\left(\begin{array}{c}
\hat{\mathbf{f}}_{\mathbf{i}} \\
0
\end{array}\right)
$$

It is composed of two terms $\hat{\mathbf{F}}_{\mathbf{u}}$ and $\hat{\mathbf{F}}_{\mathbf{i}}$, both acting as forcing terms in the momentum equations, and both related to the presence of the control cylinder in the flow.

- The first one, that reads

$$
\hat{\mathbf{f}}_{\mathbf{u}}(x, y)=-\frac{1}{2}\left(\frac{\mathbf{u}_{\mathbf{0 0}} \cdot \hat{\mathbf{u}}_{\mathbf{1 0}}^{\mathbf{c}}}{\left\|\mathbf{u}_{\mathbf{0 0}}\right\|} \mathbf{u}_{\mathbf{0 0}}+\left\|\mathbf{u}_{\mathbf{0 0}}\right\| \hat{\mathbf{u}}_{\mathbf{1 0}}^{\mathbf{c}}\right) \delta\left(x-x_{f}, y-y_{f}\right),
$$

is the spatial structure of the unsteady component of the force $\mathbf{f}_{\mathbf{u}}=\hat{\mathbf{f}}_{\mathbf{u}}(x, y) \exp \left[i \omega_{c} t\right]+$ c.c. modeling the presence of the control cylinder (see Eq. 6).

- The second one, that reads

$$
\hat{\mathbf{f}}_{\mathbf{i}}(x, y)=-\nabla \hat{\mathbf{u}}_{10}^{\mathbf{c}} \cdot \mathbf{u}_{01}-\nabla \mathbf{u}_{01} \cdot \hat{\mathbf{u}}_{10}^{\mathbf{c}},
$$

expresses the interaction of the marginal global mode $\hat{\mathbf{u}}_{10}^{\mathrm{c}}$ with the base-flow modification $\mathbf{u}_{\mathbf{0 1}}$. This term is indirectly related to the force modeling the presence of the control cylinder since the base-flow modification results from the steady component of the force through the equation (15).

We seek a form for $\mathbf{U}_{\mathbf{1 1}}$ that matches the oscillating structure of the forcing term, i.e.

$$
\mathbf{U}_{11}=\frac{1}{2}\left(\hat{\mathbf{U}}_{11} \exp \left[i \omega_{c} t\right]+c . c .\right)
$$

Introducing this decomposition into the equations (16), one obtains

$$
\left(i \omega_{c} \mathcal{L}+\mathcal{M}\right) \hat{\mathbf{U}}_{\mathbf{1 1}}=-\frac{\partial A}{\partial t_{1}} \mathcal{L} \hat{\mathbf{U}}_{\mathbf{1 0}}+A\left(\hat{\mathbf{F}}_{\mathbf{u}}+\hat{\mathbf{F}}_{\mathbf{i}}\right)
$$

The linear operator on the left-hand side of this equation is degenerated and a compatibility condition has to be satisfied to determine its solution. To this end, the following scalar product is introduced

$$
\langle\mathbf{A}, \mathbf{B}\rangle=\int_{\Omega} \mathbf{A}^{H} \mathbf{B}
$$

where ${ }^{H}$ denotes the transconjugate operator. Furthermore, one defines the adjoint global mode $\hat{\mathbf{U}}_{\mathbf{1 0}}^{\mathbf{c}+}=\left(\hat{\mathbf{u}}_{\mathbf{1 0}}^{\mathbf{c}+}, \hat{p}_{10}^{c+}\right)^{T}$ that satisfies the adjoint equation

$$
\left(-i \omega_{c} \mathcal{L}+\mathcal{M}^{+}\right) \hat{\mathbf{U}}_{10}^{\mathbf{c}+}=0
$$

where the operator $\mathcal{M}^{+}$is defined by

$$
\mathcal{M}^{+}=\left(\begin{array}{cc}
-\nabla() \cdot \mathbf{u}_{\mathbf{0 0}}+\left(\nabla \mathbf{u}_{\mathbf{0 0}}\right)^{T} \cdot()-R e_{c}^{-1} \Delta & -\nabla \\
\nabla^{T} & 0
\end{array}\right)
$$

By multiplying (in the sense of the above scalar product) the equation (21) with the adjoint global mode, one obtains the compatibility condition:

$$
\frac{\partial A}{\partial t_{1}}=C_{1} A\left(t_{1}\right), \text { with } C_{1}=\left\langle\hat{\mathbf{U}}_{\mathbf{1 0}}^{\mathbf{c}+}, \hat{\mathbf{F}}_{\mathbf{u}}+\hat{\mathbf{F}}_{\mathbf{i}}\right\rangle
$$


Note that the adjoint global modes is normalized so that $\left\langle\hat{\mathbf{U}}_{\mathbf{1 0}}^{\mathbf{c}+}, \mathcal{L} \hat{\mathbf{U}}_{\mathbf{1 0}}^{\mathbf{c}}\right\rangle=1$. The amplitude of the perturbation is thus $A(t)=\exp \left[\left(\epsilon C_{1}\right) t\right]$ and the perturbation reads

$$
\mathbf{U}_{\mathbf{1 0}}=\frac{1}{2}\left(\hat{\mathbf{U}}_{\mathbf{1 0}}^{\mathbf{c}} \exp \left[\sigma_{f} t\right]+\text { c.c. }\right), \text { with } \sigma_{f}=\sigma_{c}+\epsilon C_{1}
$$

The growth rate $\lambda_{f}=\Re\left(\sigma_{f}\right)$ and pulsation $\omega_{f}=\Im\left(\sigma_{f}\right)$ of the perturbation developing when a control cylinder is placed into the flow are given by

$$
\begin{aligned}
\lambda_{f} & =\epsilon \Re\left(C_{1}\right) \\
\omega_{f} / \omega_{c} & =1+\epsilon \Im\left(C_{1}\right) / \omega_{c}
\end{aligned}
$$

These two relations are fundamental to determine the impact of a control cylinder on the flow stability. In particular, the control cylinder stabilizes the flow if $\lambda_{f}=\epsilon \Re\left(C_{1}\right)<0$ and destabilizes it if $\lambda_{f}=$ $\epsilon \Re\left(C_{1}\right)>0$. The major contribution of the multiple timescale analysis developed in this paper is that the influence of the steady and unsteady components of the force modeling the control cylinder are both taken into account and can be analysed separately. To this end, the complex eigenvalue $\sigma_{f}$ is written as

$$
\sigma_{f}=\sigma_{c}+\sigma_{f, s}+\sigma_{f, u}
$$

where $\sigma_{f, s}$ and $\sigma_{f, u}$ are the contributions due to, respectively, the steady and unsteady components of the force. These two contribution read:

$$
\begin{aligned}
\sigma_{f, s} & =\epsilon\left\langle\hat{\mathbf{U}}_{\mathbf{1 0}}^{\mathbf{c}+}, \hat{\mathbf{F}}_{\mathbf{i}}\right\rangle \\
\sigma_{f, u} & =\epsilon\left\langle\hat{\mathbf{U}}_{\mathbf{1 0}}^{\mathbf{c}+}, \hat{\mathbf{F}}_{\mathbf{u}}\right\rangle
\end{aligned}
$$

First, let us examine the unsteady contribution $\sigma_{f, u}$. In the above expression the unsteady component $\hat{\mathbf{F}}_{\mathbf{u}}$ appear explicitely and the adjoint global mode plays the role of a sensitivity function with respect to this component of the force. It is thus straightforward to obtain the values of $\sigma_{f, u}$ for all positions of the force $\left(x_{f}, y_{f}\right)$ by computing the adjoint global mode $\hat{\mathbf{U}}_{\mathbf{1 0}}^{\mathbf{c}+}$ (Eq.23) and the unsteady component (Eq.18).

Now, let us examine the steady contribution $\sigma_{f, s}$. The steady component $\hat{\mathbf{F}}_{\mathbf{s}}$ does not appear explicitely but only implicitely through the term $\hat{\mathbf{F}}_{\mathbf{i}}$, as explained previously (see Eq. 19). Thus, in the present form, it is not straightforward to obtain the values of $\sigma_{f, s}$ for all positions $\left(x_{f}, y_{f}\right)$, since it requires to solve the equation (13) for all positions of the steady force (Eq. 14). The next paragraph explains how to overcome this drawback by introducing a sensitivity function with respect to the steady component of the force.

\section{Sensitivity analysis}

The adjoint global mode appears naturally in the multiple timescale analysis. It has been shown that, for the eigenvalue, it plays the role of a sensitivity function with respect to the unsteady component of the force modeling the control cylinder. For a more general discussion about the use of adjoint global modes as receptivity and sensitivity functions, the reader is refered to Giannetti and Luchini (2007). Marquet et al. (2008) have recently introduced a sensitivity function of a global eigenvalue with respect to a steady force. In the present case, it is useful to introduce such a sensitivity function, denoted $\mathbf{U}_{\mathbf{0 0}}^{+}$, in order to relate explicitely the steady contribution $\sigma_{f, s}$ to the steady component of the force $\hat{\mathbf{F}}_{\mathbf{s}}$. This is written

$$
\sigma_{f, s}=\epsilon\left\langle\mathbf{U}_{\mathbf{0 0}}^{+}, \hat{\mathbf{F}}_{\mathbf{s}}\right\rangle
$$

where the complex flowfield $\mathbf{U}_{\mathbf{0 0}}^{+}$satisfies the so-called adjoint base-flow equation:

$$
\mathcal{M}^{+} \mathbf{U}_{\mathbf{0 0}}^{+}=\left(\begin{array}{c}
\left(\nabla \hat{\mathbf{u}}_{\mathbf{1 0}}^{\mathbf{c}+}\right) \hat{\mathbf{u}}_{\mathbf{1 0}}^{\mathbf{c} *}-\left(\nabla \hat{\mathbf{u}}_{\mathbf{1 0}}^{\mathbf{c}}\right)^{t} \hat{\mathbf{u}}_{\mathbf{1 0}}^{\mathbf{c}+} \\
0
\end{array}\right)
$$


The procedure to derive this equation is detailed in Appendix. This equation is linear and the right-hand side term is a forcing term that involves the global mode velocity $\hat{\mathbf{u}}_{10}^{\mathrm{c}}$ and the adjoint global mode velocity $\hat{\mathbf{u}}_{\mathbf{1 0}}^{\mathbf{c}+}$. An important point to notice is that the sensitivity function, solution of this equation, does not depend on the position of the force $\left(x_{f}, y_{f}\right)$. Consequently, it is now straightforward to obtain the values of $\sigma_{f, s}$ for all positions of the force $\left(x_{f}, y_{f}\right)$ by using Eq.(32) instead of Eq.(30). It only requires to solve the adjoint base-flow equations (33) and to compute the steady component of the force.

Finally, since the force is localized at the point $\left(x_{f}, y_{f}\right)$, the steady $\sigma_{f, s}$ and unsteady contribution $\sigma_{f, u}$ read:

$$
\begin{aligned}
\sigma_{f, s}\left(x_{f}, y_{f}\right) & =\epsilon\left(x_{f}, y_{f}\right) \mathbf{u}_{\mathbf{0 0}}^{+*}\left(x_{f}, y_{f}\right) \hat{\mathbf{f}}_{\mathbf{s}}\left(x_{f}, y_{f}\right) \\
\sigma_{f, u}\left(x_{f}, y_{f}\right) & =\epsilon\left(x_{f}, y_{f}\right) \hat{\mathbf{u}}_{\mathbf{1 0}}^{\mathbf{c}+*}\left(x_{f}, y_{f}\right) \hat{\mathbf{f}}_{\mathbf{u}}\left(x_{f}, y_{f}\right)
\end{aligned}
$$

These two relations give the impact of the control cylinder on the flow stability.

\section{Results for the cylinder flow}

The incompressible flow around a cylinder is studied for low values of the Reynolds number. At the threshold $R e \sim 47$, it is known that the two-dimensional flow bifurcates from a steady symetric state towards a periodic non-symetric state. In this section, the cylinder flow (without control) is first briefly described in the framework of a global stability analysis. The theoretical developed in the previous section is then applied to predict accurately the positions of the control cylinder that stabilize the flow.

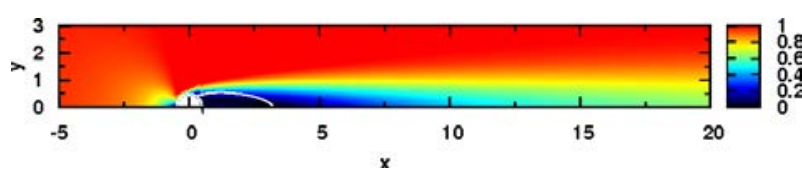

(a) Base Flow

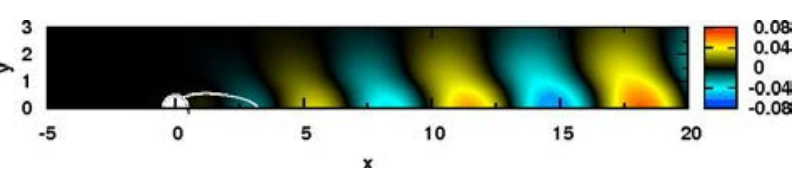

(b) Neutral mode

Figure 1. Base-flow and neutral global mode at the critical Reynolds number $\boldsymbol{R e}_{c}=46.8$ depicted in the upper-half plane $y>0$. (a) Magnitude of the base-flow velocity $\left\|\mathbf{u}_{00}\right\|$ (b) Real part of the vertical velocity of the neutral mode $\Re\left(\hat{v}_{10}\right)$. The white curve depicts the divided streamlines of the recirculation region.

\section{A. Cylinder flow and global stability analysis}

The steady symetric state of the cylinder flow, i.e. the base-flow, is depicted in figure 1(a) for the Reynolds number $R e=46.8$. As expected, a recirculation region, delimited in the figure by the divided streamline (white curve), develops in the near-wake of the cylinder. Performing a global stability analysis of such a baseflow is an efficient approach to determine for which Reynolds number values this flow state remains observable. Such an analysis has first been performed by Zebib $(1987)^{8}$ but many authors have then considered this problem. Figure 2 depicts the growth rate $\lambda$ and the pulsation $\omega$ of the leading global mode as a function of the Reynolds number. The critical Reynolds number $R e_{c}=46.8$, outlined by the vertical dashed line, is determined as the value of $R e$ for which the flow is marginally stable, i.e. $\lambda_{c}=\lambda\left(R e_{c}\right)=0$. The critical pulsation of the global mode is $\omega_{c}=0.73$. The leading global mode at this Reynolds number is called the neutral mode. The real part of its vertical velocity is depicted in figure 1(b). It represents an array of counter-rotating vortices which develop in the wake of the cylinder, very similar to the Von-Karman street observed experimentally. Note that maximal values of the neutral mode are reached in the far-wake of the cylinder $(x \sim 25$, not shown here).

\section{B. Passive control of the cylinder flow}

The passive control of the Von-Karman street by means of a smaller control cylinder of diameter $d \leq D$ has been experimentaly studied by Strykowski \& Sreenivasan (1990). Figure 3 reproduces their results obtained for the cylinder ratio $d / D=0.1$. Each curve is associated to a Reynolds number $R e>R e_{c}$. Within this curve the placement of a control cylinder suppresses the Von-Karman street behind the main cylinder. Interestingly, the stabilizing regions, thus delimited, are located close the main cylinder, and not 

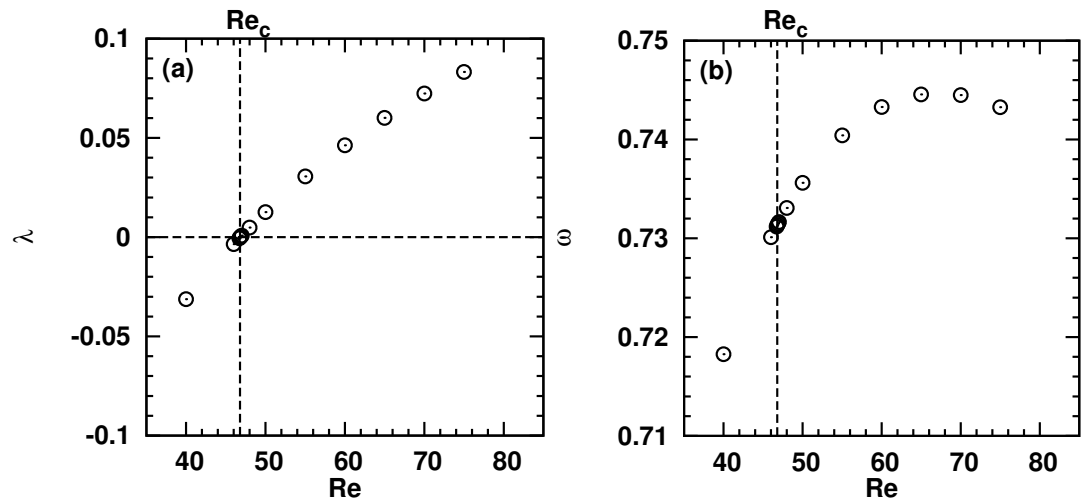

Figure 2. Global stability of the cylinder flow (without control). (a) Growth rate $\lambda$ and (b) pulsation $\omega$ (bottom) of the leading global mode as a function of the Reynolds number Re. The vertical dashed line outlines the threshold of the flow instability at $\boldsymbol{R e}_{c}=46.8$. The pulsation at the criticity is $\omega_{c} \sim 0.73$.

in the far wake region where the amplitude of the global mode is maximal. This suggests, as already noted by Chomaz $(2005)^{1}$ and Luchini \& Giannetti $(2003),{ }^{2}$ that the region of the flow responsible for the onset of the instability, i.e. the wavemaker, can not be identified by examining the structure of the unstable global mode. For further details about the definition of the wavemaker, the reader is refered to Giannetti \& Luchini $(2007)^{3}$ who have proposed a general method to identify this wavemaker region. The theoretical approach developed in the previous section is now applied to the cylinder flow. The objective is not to reproduce quantitatively the experimental results of Strykowski \& Sreenivasan $(1990)^{7}$ since the present approach is only valid at the critical Reynolds number $R e_{c}$ (in its present state). The main issue is more to demonstrate that a modelisation of the control cylinder by a force should include both the steady and unsteady components of the force to obtain accurate results.

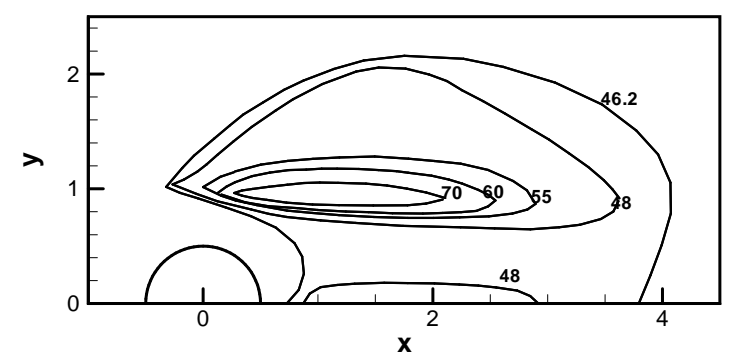

Figure 3. Experimental results by Strykowski \& Sreenivasan (1990).

\section{Steady and unsteady components of the force}

The local force that models the presence of the control cylinder located at the station $\left(x_{f}, y_{f}\right)$ has two components (see Eq.6): a steady component $\mathbf{f}_{\mathbf{s}}$, that depends only on the base-flow velocity, and an unsteady component $\mathbf{f}_{\mathbf{u}}=\hat{\mathbf{f}}_{\mathbf{u}} \exp \left[i_{c} t\right]$, that depends on both the base-flow and global mode velocities. The magnitude of these two components are respectively depicted in figure 4(a) and figure 4(b) as a function of the station $\left(x_{f}, y_{f}\right)$ of the force. It shows that the magnitude of the steady component is one of order larger than the magnitude of the unsteady component. Highest magnitude of the steady and unsteady forces are reached in differents regions of the flow. For the steady component (figure 4(a)), largest values are reached in regions where the base-flow velocity is large. Thus, in the recirculation region where the base-flow velocity is weak, the magnitude of the steady component is also weak. For the unsteady component (figure 4(b)), largest values are reached in the far-wake of the cylinder $(x \sim 20)$, the region where the magnitude of the global mode velocity reaches highest values. 


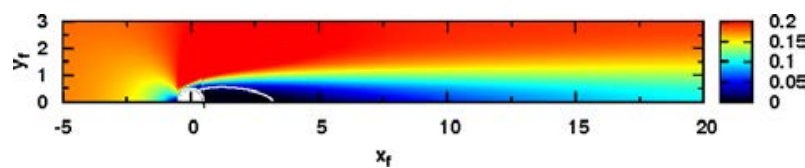

(a) $\left\|\hat{\mathbf{f}}_{\mathbf{s}}\left(x_{f}, y_{f}\right)\right\|$

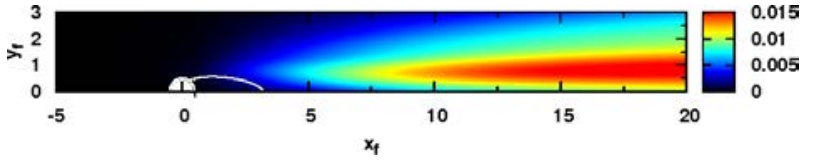

(b) $\left\|\hat{\mathbf{f}}_{\mathbf{u}}\left(x_{f}, y_{f}\right)\right\|$

Figure 4. Local force modeling the control cylinder. Amplitude of the (a) steady and (b) unsteady components of this force as a function of its location $\left(x_{f}, y_{f}\right)$. Parameter: $\boldsymbol{R e}=\boldsymbol{R} \boldsymbol{e}_{c}=46.8$.

\section{Sensitivities of the neutral global mode}

The sensitivity of the neutral global mode to a steady and an unsteady force is now examined. We recall that the sensitivity to a steady force is given by the adjoint base-flow velocity, whereas the sensitivity to an unsteady force is given by the adjoint global mode velocity. The magnitude of these two sensitivities functions is depicted in figure 5(a) and 5(b). First, it shows that the neutral mode is more sensitive to an

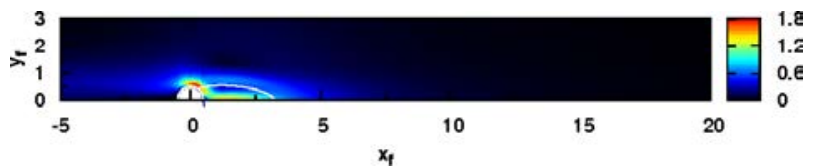

(a) $\left\|\hat{\mathbf{u}}_{\mathbf{0 0}}^{+}(x, y)\right\| ;$ Adjoint base-flow

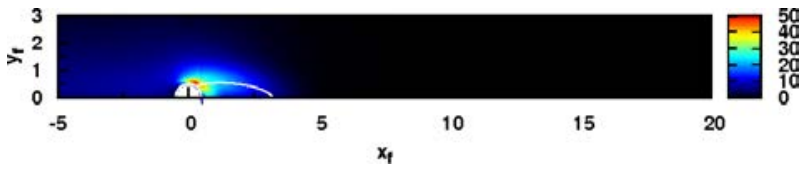

(b) $\left\|\hat{\mathbf{u}}_{10}^{+}(x, y)\right\|$; Adjoint global mode

Figure 5. Sensitivity functions of the neutral global mode. Magnitude of (a) the sensitivity to a steady force and of (b) the sensitivity to an unsteady force in the plane $(x, y)$. Parameter: $\boldsymbol{R} e=\boldsymbol{R}_{c}=46.8$.

unsteady force than to a steady force, since the magnitude of the sensitivity function is one of order larger in figure 5(b) than in figure 5(a). Secondly, we observe that, far from the cylinder, the neutral mode is neither sensitivite to a steady nor to an unsteady force. Indeed, the sensitivity regions are located close the cylinder in the two figures. The region close to the separation point is sensitivite both to a steady and an unsteady force. Figure 5(a) shows that the recirculation region is a sensitivite region fo the steady force.

Given the relation (34), the control cylinder should have a large impact onto the flow stability only if it is placed in the overlapping region between the components of the force and the sensitivity functions, i.e. the region where the product of the force and sensitivity magnitudes is large. By comparing figures 4(a)-4(b) with figures 5(a)-5(b), we expect that, for the cylinder flow, this overlapping region is in the near-wake of the cylinder.

\section{Growth rate $\lambda_{f}$}

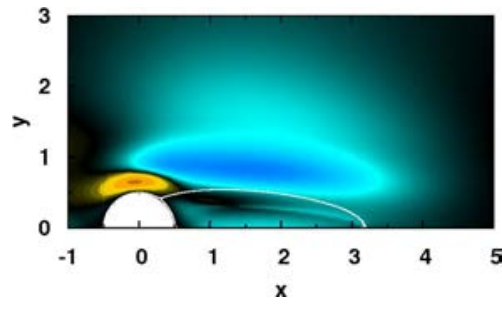

(a) $\lambda_{f}=\lambda_{f, s}+\lambda_{f, u}$

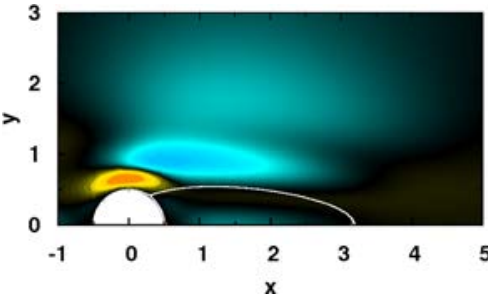

(b) $\lambda_{f, s}$

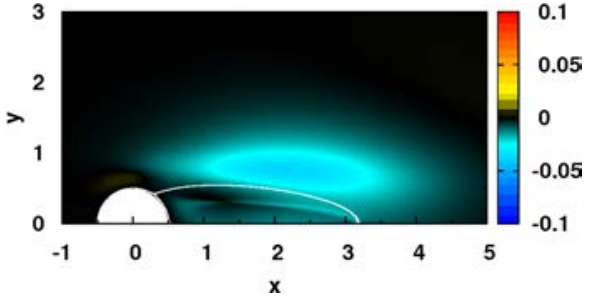

(c) $\lambda_{f, u}$

Figure 6. Growth rate of the neutral mode as a function of the location of the force $\left(x_{f}, y_{f}\right)$. The growth rate (a) $\lambda_{f}$ is the sum of the two contributions, (b) $\lambda_{f, s}$ and (c) $\lambda_{f, u}$, induced by, respectively, the steady and unsteady components of the force. The same colorbar is used in the three figures. Parameter: $R e=R e_{c}=46.8$.

In this paragraph we focus on the growth rate $\lambda_{f}$. We look for the locations of the force that stabilize $\left(\lambda_{f}<0\right)$ or destabilize $\left(\lambda_{f}>0\right)$ the flow. Figure 6(a) depicts $\lambda_{f}$ in the half-plane $\left(x_{f}, y_{f}>0\right)$. It shows a large stabilizing region located above the recirculation region and a smaller one located inside the recirculation region. Close to the cylinder and upstream from the separation point, there exists a destabilizing region. 
The contributions of the steady and unsteady components of the force are respectively plotted in figures $6(\mathrm{~b})$ and 6(c). First we see that the two contributions are of the same order of magnitude. Therefore, the contribution of one of these components can not be neglected compare to the other one. Secondly, the two contributions have approximatively the same signs in the same regions. It means that the steady and unsteady components of the force have a cooperative effect on the stabilization of the flow.

\section{Pulsation $\omega_{f}$}

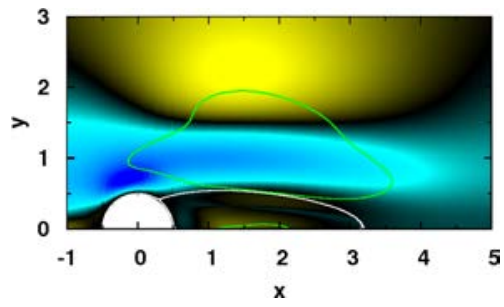

(a) $\omega_{f} / \omega_{c}=1+\left(\omega_{f, s}+\omega_{f, u}\right) / \omega_{c}$

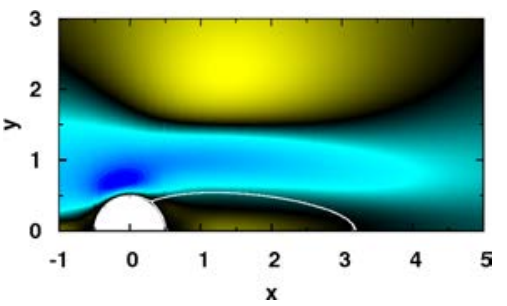

(b) $1+\omega_{f, s} / \omega_{c}$

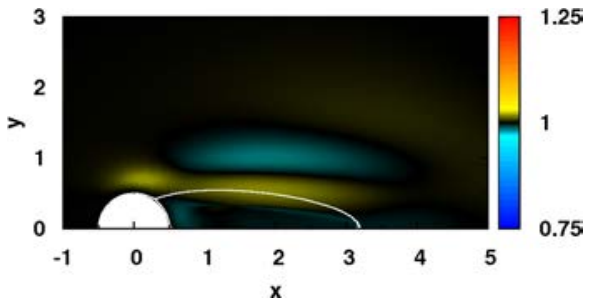

(c) $1+\omega_{f, u} / \omega_{c}$

Figure 7. Same caption as for the previous figure, but for the pulsation ratio $\omega_{f} / \omega_{c}$.

In this paragraph we examine the dependance of the pulsation $\omega_{f}$ with the position of the force. Figure 7 (a) depicts $\omega_{f} / \omega_{c}$, the ratio of the pulsation with control over the pulsation without control, in the halfplane $y_{f}>0$. For $\omega_{f} / \omega_{c}=1$, the control cylinder does not modify the pulsation. For $\omega_{f} / \omega_{c}>1$, the control cylinder increases the pulsation, and inversely when $\omega_{f} / \omega_{c}<1$. In the figure, the green line delimit approximatively the stabilizing regions determined in the previous paragraph. Placing a control cylinder in these regions stabilizes the flow. Thus it is not relevant to examine the pulsation in these regions. We focus our attention on the value of ratio $\omega_{f} / \omega_{c}$ obtained out of the stabilizing regions. Figure 7 (a) clearly shows that the largest decrease of the pulsation can be obtained if the control cylinder is placed close to the cylinder, upstream from the separation point. In figures $7(\mathrm{~b})$ and $7(\mathrm{~b})$ are depicted the contributions of the steady and unsteady components of the force. Strikingly, it shows that the dominant contribution is the one induced by the steady component. We note that the results obtained for the pulsation should be considered with caution. Indeed, it is well known that, for the cylinder flow without passive control, a global linear stability analysis fails to predict accurately the pulsation of the Von-Karman street that is measured in experiments. In particular, it has been recognised that, during the saturation process of the linear instability, the non-linearities tends to strongly modify the frequency. We may expect that the non-linearities also strongly modifies the pulsation instability when the flow is controlled.

\section{Conclusion}

This paper is a theoretical contribution to the passive control of globally unstable flows. It is based on the assumption that introducing a small control device into the flow can be modeled by a local force. Up to now, the effect of such a force onto the flow stability has been alleviated by assuming that it was either a steady or an unsteady force. The formalism developed in this paper enables to model the force as the sum of a steady and an unsteady component, and to take into account their respective effects onto the flow stability. By applying this approach to the passive control of the cylinder flow, it has been shown that both the steady and unsteady components of the force affect the flow stability. This result suggests that a modelisation of the control cylinder as the sum of a steady and unsteady force is essential to obtain a good prediction of the stabilizing regions. The main drawback of the present formalism is that the passive control of the flow can be investigated only at the critical Reynolds number $R e_{c}$. Future developments should be dedicated to extent the range of Reynolds number for which this approach can be applied. Introducing a third parameter, in the multiple timescale analysis, which measures the departure from criticality (in terms of the Reynolds number) seems to be an interesting way to explore. 


\section{Appendix}

In this Appendix, it is explained how the sensitivity function to a steady force is introduced and how the adjoint base-flow equations are derived. The idea is to make appear explicitely the steady component of the force $\mathbf{F}_{\mathbf{s}}$ in the expression of $\sigma_{f, s}$. To this end we introduce the operator $\mathcal{L}_{r}=\left(\begin{array}{ll}\mathcal{I} & 0\end{array}\right)$ that satisfies the following relations

$$
\mathcal{L}_{r}^{T} \mathcal{L}_{r}=\mathcal{L}, \quad \mathcal{L}_{r} \mathcal{L}_{r}^{T}=\mathcal{I}
$$

The velocity $\mathbf{u}_{\mathbf{0 1}}$ can thus be related to the flowfield variable $\mathbf{U}_{\mathbf{0 1 0}}$ through

$$
\mathbf{u}_{01}=\mathcal{L}_{r} \mathbf{U}_{01}
$$

Using the definition of $\hat{\mathbf{F}}_{\mathbf{i}}$ (Eq.17) and the equation (13) to express the base-flow modification $\mathbf{U}_{\mathbf{0 1}}$ as a function of the steady force $\mathbf{F}_{\mathbf{s}}$, one writes

$$
\begin{aligned}
\left\langle\hat{\mathbf{U}}_{\mathbf{1 0}}^{\mathrm{c}+}, \hat{\mathbf{F}}_{\mathbf{i}}\right\rangle= & -\left\langle\hat{\mathbf{u}}_{\mathbf{1 0}}^{\mathrm{c}+}, \nabla \hat{\mathbf{u}}_{\mathbf{1 0}}^{\mathrm{c}} \cdot \mathbf{u}_{\mathbf{0 1}}+\nabla \mathbf{u}_{\mathbf{0 1}} \cdot \hat{\mathbf{u}}_{\mathbf{1 0}}^{\mathrm{c}}\right\rangle \\
= & -\left\langle\hat{\mathbf{u}}_{\mathbf{1 0}}^{\mathrm{c}+}, \nabla \hat{\mathbf{u}}_{\mathbf{1 0}}^{\mathrm{c}} \cdot\left(\mathcal{L}_{r} \mathbf{U}_{\mathbf{0 1}}\right)+\nabla\left(\mathcal{L}_{r} \mathbf{U}_{\mathbf{0 1}}\right) \cdot \hat{\mathbf{u}}_{\mathbf{1 0}}^{\mathrm{c}}\right\rangle \\
= & -\left\langle\hat{\mathbf{u}}_{\mathbf{1 0}}^{\mathrm{c}+}, \nabla \hat{\mathbf{u}}_{\mathbf{1 0}}^{\mathrm{c}} \cdot\left(\mathcal{L}_{r} \mathcal{M}^{-1} \mathbf{F}_{\mathbf{s}}\right)+\nabla\left(\mathcal{L}_{r} \mathcal{M}^{-1} \mathbf{F}_{\mathbf{s}}\right) \cdot \hat{\mathbf{u}}_{\mathbf{1 0}}^{\mathrm{c}}\right\rangle
\end{aligned}
$$

Performing the following integrations by parts

$$
\begin{aligned}
& \left\langle\hat{\mathbf{u}}_{10}^{\mathbf{c}+}, \nabla \hat{\mathbf{u}}_{\mathbf{1 0}}^{\mathbf{c}} \cdot\left(\mathcal{L}_{r} \mathcal{M}^{-1} \mathbf{F}_{\mathbf{s}}\right)\right\rangle=\left\langle\left(\mathcal{M}^{-1}\right)^{H} \mathcal{L}_{r}^{T}\left(\nabla \hat{\mathbf{u}}_{10}^{\mathbf{c} *}\right)^{T} \hat{\mathbf{u}}_{10}^{\mathbf{c}+}, \mathbf{F}_{\mathbf{s}}\right\rangle \\
& \left\langle\hat{\mathbf{u}}_{\mathbf{1 0}}^{\mathbf{c}+}, \nabla\left(\mathcal{L}_{r} \mathcal{M}^{-1} \mathbf{F}_{\mathbf{s}}\right) \cdot \hat{\mathbf{u}}_{\mathbf{1 0}}^{\mathbf{c}}\right\rangle=\left\langle-\left(\mathcal{M}^{-1}\right)^{H} \mathcal{L}_{r}^{T}\left(\nabla \hat{\mathbf{u}}_{10}^{\mathbf{c}+}\right) \hat{\mathbf{u}}_{10}^{\mathbf{c} *}, \mathbf{F}_{\mathbf{s}}\right\rangle
\end{aligned}
$$

one obtains

$$
\left\langle\hat{\mathbf{U}}_{\mathbf{1 0}}^{\mathbf{c}+}, \hat{\mathbf{F}}_{\mathbf{i}}\right\rangle=\left\langle\left(\mathcal{M}^{-1}\right)^{H} \mathcal{L}_{r}^{T}\left\{\left(\nabla \hat{\mathbf{u}}_{\mathbf{1 0}}^{\mathbf{c}+}\right) \hat{\mathbf{u}}_{\mathbf{1 0}}^{\mathbf{c *}}-\left(\nabla \hat{\mathbf{u}}_{\mathbf{1 0}}^{\mathbf{c} *}\right)^{T} \hat{\mathbf{u}}_{\mathbf{1 0}}^{\mathbf{c}+}\right\}, \mathbf{F}_{\mathbf{s}}\right\rangle
$$

One introduces the variable $\mathbf{U}_{\mathbf{0 0}}^{+}=\left(\mathbf{u}_{\mathbf{0 0}}^{+}, p_{00}^{+}\right)$, called the adjoint of the base-flow and enforce it to satisfies the adjoint base-flow equations

$$
\mathcal{M}^{H} \mathbf{U}_{\mathbf{0 0}}^{+}=\mathcal{L}_{r}^{T}\left\{\left(\nabla \hat{\mathbf{u}}_{\mathbf{1 0}}^{\mathbf{c}+}\right) \hat{\mathbf{u}}_{\mathbf{1 0}}^{\mathbf{c} *}-\left(\nabla \hat{\mathbf{u}}_{\mathbf{1 0}}^{\mathbf{c} *}\right)^{T} \hat{\mathbf{u}}_{\mathbf{1 0}}^{\mathbf{c}+}\right\}
$$

Thus one obtains that

$$
\left\langle\hat{\mathbf{U}}_{\mathbf{1 0}}^{\mathrm{c}+}, \hat{\mathbf{F}}_{\mathbf{i}}\right\rangle=\left\langle\mathbf{U}_{\mathbf{0 0}}^{+}, \mathbf{F}_{\mathbf{s}}\right\rangle
$$

\section{References}

\footnotetext{
${ }^{1}$ Chomaz J.M., Global instabilities in spatially developping flows: non-normality and nonlinearity, Annu. Rev. Fluid Mech., $37,357-392,2005$.

${ }^{2}$ Giannetti F. \& Luchini P., Receptivity of the circular cylinder's first instability, Proc. $5^{\text {th }}$ Eur. Fluid Mech. Conf., Toulouse,

${ }^{3}$ Giannetti F. \& Luchini P., Structural sensitivity of the cylinder wake's first instability, J. Fluid Mech., 581, 167-197, 2007.

${ }^{4}$ Hill D.C., A Theoretical Approach for Analysing the Restabilization of Wakes, AIAA, 92-0067, 30th Aerospace Sciences Meeting \& Exhibit, January, 1992, Reno.

${ }^{5}$ Marquet O., Sipp D. \& Jacquin L., Sensitivity analysis and passive control of the cylinder flow, Submitted to J. Fluid

${ }^{6}$ Sipp D. \& Lebedev A., Global stability of base- and mean-flows: a general approach and its applications to cylinder and open cavity flows, J. Fluid Mech., 593, 333-358, 2007.

${ }^{7}$ Strykowski P.J. \& Sreenivasan K.R., On the formation and suppression of vortex shedding at 'low' Reynolds numbers, J. Fluid Mech., 218, 71-107, 1990.

${ }^{8}$ Zebib, A., Stability of viscous flow past a circular cylinder, J. Engrg Math., 21, 155-165, 1987.
} 2003. Mech. 